\title{
HIGH LATITUDE PLASMA CONVECTION: PREDICTIONS FOR EISCAT AND SONDRE STROMFJORD
}

\section{J.J. Sojka, W.J. Raitt and R.W. Schunk}

Center for Atmospheric and Space Sciences, Utah State University, Logan, Utah 84322

\begin{abstract}
We have used a plasmo convection model to predict diurnal patterns of horizontal drift velocities in the vicinity of the EISCAT incoherent scatter facility at Tromso, Norway and for Sondre Stromfjord, Greenland, a proposed new incoherent scatter facility site. The convection model includes the offset of $11.4^{\circ}$ between the geographic and geomagnetic poles (northern hemisphere), the tendency of plasma to corotate about the geographic pole, and a magnetospheric electric field mapped to a circle about a center offset by $5^{\circ}$ in the antisunward direction from the magnetic pole. Four different magnetospheric electric field configurations were considered, including a constant cross- tail electric field, asymmetric electric fields with enhancements on the dawn and dusk sides of the polar cap, and an electric field pattern that is not aligned parallel to the noon-midnight magnetic meridian. The different electric field configurations produce different signatures in the plasma convection pattern which are clearly identified. Both of these high-latitude sites are better suited to study magnetospheric convection effects than either Chatanika, Alaska or Millstone Hill, Massachusetts. Also, each site appears to have unique capabilities with regard to studying certain aspects of the magnetospheric electric field.
\end{abstract}

\section{Introduction}

The measurement of ionospheric plasma convection by the ground-based, incoherent scatter technique has an advantage over satellite measurements of plasma velocities in that a wide range of latitudes and all local times can be covered during the course of one day. During periods of relatively constant activity incoherent scatter measurements allow the observation of features like the Harang discontinuity, the polar cap boundary, and regions of flow reversal. Even for relatively simple magnetospheric electric field models the resulting plasma convection pattern observed in a geographic frame of reference is rather complex and universal time (UT) dependent. Sojka et al. (1979a) modelled the result of mapping a dawn-dusk, cross-tail magnetospheric electric field into the ionosphere about the geomagnetic pole, which was offset by $11.4^{\circ}$ from the geographic pole. In addition, the tendency of the ionospheric plasma to corotate about the geographic pole was included.

In order to determine the extent to which our simple convection model could account for specific observations, plasma convection patterns predicted by our model were compared to those observed at Chatanika, Alaska and Millstone Hill, Massachusetts (Sojka et al., 1979b). These two incoherent scatter facilities operated simultaneously for four days in June, 1978 to provide data sets which were averaged to 24 hours in order to minimize the effects of individual substorms. As a result of the comparison an additional feature was added to the model in order to improve the agreement with the data. Instead of being mapped about the geomagnetic pole, the magnetospheric electric field was mapped about a point $5^{\circ}$ along the midnight magnetic meridian from the geomagnetic pole. With this modification good agreement was obtained between our simple convection model and the different diurnal patterns observed simultaneously at Chatanika and Millstone Hill.

Although good agreement was found for the data sets chosen, the latitudinal range over which data were available is restrictive as far as some of the features associated with the Copyright 1979 by the American Geophysical Union. magnetospheric electric field model are concerned. At least three additional features predicted by our model need further investigation, but cannot adequately be studied from either Chatanika or Millstone Hill; these are: (i) Latitude of the midnight polar boundary and the flow pattern inside the midnight polar region; (ii) Local times of the noon and midnight flow direction reversals; (iii) Latitude of the noon polar boundary and the flow pattern inside the noon polar region.

In order to gain further insight into the effects of magnetospheric convection, we calculated plasma convection patterns which would be observed by the European Incoherent Scatter Radar System (EISCAT) and by a radar site located at Sondre Stromfjord, Greenland. EISCAT is due to become operational in the near future and Sondre Stromfjord has recently been suggested as a possible site for another high-latitude incoherent scatter radar system. For both sites plasma convection patterns were calculated for several magnetospheric electric field configurations, including a constant cross-tail electric field, asymmetric electric fields with enhancements on the dawn and dusk sides, and an electric field pattern that is not aligned parallel to the noon-midnight magnetic meridian. Each site appears to have unique capabilities. EISCAT with its better sensitivity and slightly higher magnetic latitude coverage will enable features (i) and (ii) in the above list to be studied. Additionally, EISCAT is approximately $180^{\circ}$ of longitude from Chatanika, which will enable a further test of the predicted UT influence on the plasma convection pattern to be made. The importance of the Sondre Stromfjord location, on the other hand, is that it allows feature (iii), the noon polar cap boundary, to be studied. Furthermore, it is the only site proposed to date from which the polar cap itself could be studied at all local times.

\section{Plasma Convection Model}

A series of four convection patterns were computed from a basic model (Sojka et al., 1979b) to which adjustments had been made to reproduce different features of the magnetospheric convection electric field. The basic convection model includes the offset of $11.4^{\circ}$ between the geographic and geomagnetic poles (Mead, 1970), the tendency of plasma to corotate about the geographic pole, and a constant dawn-dusk magnetospheric electric field mapped to a circle about a center offset by $5^{\circ}$ in the anti-sunward direction from the magnetic pole. The radius of the circle corresponds to $17^{\circ}$ of latitude and the electric potentials are aligned parallel to the noon/midnight meridian within the circle. Equatorward of the circle the potential diminishes meridionally and inversely as the fourth power of sine magnetic co-latitude. Figure 1 (model a) shows the potential distribution for this magnetospheric electric field. Magnetic local time in hours and magnetic latitude at $10^{\circ}$ intervals are shown in the figure. Contours of potential have been computed at 5 kilovolt intervals; five of these contours have been labelled. A total potential drop of 90 kilovolts was used in this and subsequent calculations. This potential is representative of moderately active magnetospheric conditions.

The second and third potential models result from adding an asymmetry to the polar cap electric field. In the first model (Figure la) the polar region contains a uniform electric field. By enhancing the dawn or dusk polar cap electric field it is possible to simulate the effects of different orientations of the interplanetary magnetic field on the magnetospheric convection elec- 


\section{MAGNETOSPHERIC POTENTIAL MODELS}

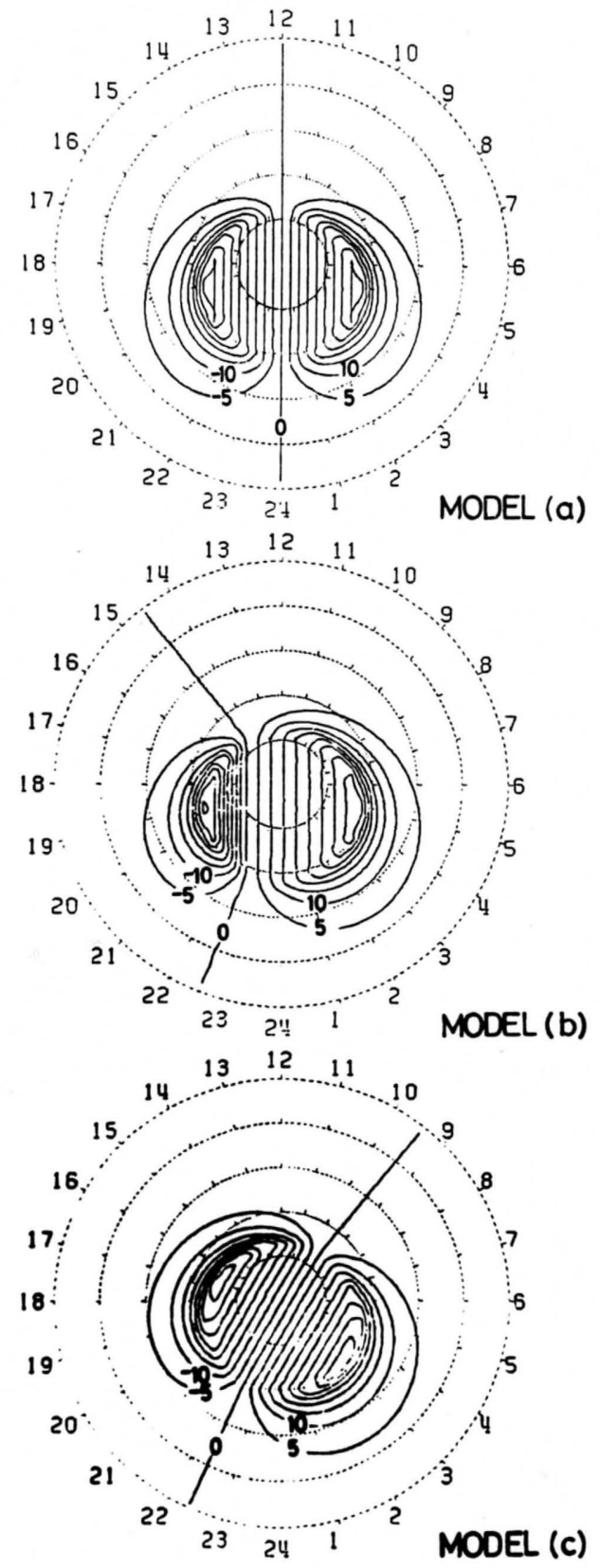

Fig. 1 Magnetospheric electric potentials mapped into the polar ionosphere shown in magnetic coordinates. Model (a) is for a uniform cross-tail electric field mapped about a point offset $5^{\circ}$ in the anti-sunward direction from the magnetic pole. Model (b) has the same characteristics as model (a), except the magnetospheric electric field is asymmetric with the enhancement oil the dusk side. Model (c) has the same characteristics as model (a), except the magnetospheric potential distribution is rotated to be parallel to the 10-22 MLT meridian. All models are for a total cross-tail potential of $90 \mathrm{kV}$ and the contour interval is $5 \mathrm{kV}$.

tric field (cf. Fairfield, 1977; Heppner, 1977; Stern, 1977). Figure 1 (model b) shows the effect of an enhanced dusk electric field on the magnetospheric potential distribution. The symmetry present in Figure 1a about the zero potential contour is no longer present; the pattern now has one small cell (dusk) and one large cell (dawn). The magnetospheric potential distribution for our enhanced dawn electric field is the mirror image of Figure lb about the noon-midnight meridian and is not shown.

The fourth model contains a rotation of the whole magnetospheric electric field by two hours around the point about which the field is mapped into the ionosphere. Empiri. cally it was found that this adjustment gave very good agree ment between the model and the finer details of magnetospheric convection as observed at Chatanika and Millstone Hill. Such a skewing has been associated with the polar cap current systems (Yasuhara et al., 1975); however, in these cases the skewing is attributed to asymmetric conductivity distributions with a symmetric electric field. Figure 1 (model c) shows the result of rotating the magnetospheric electric field of Figure 1a by two hours such that the zero potential contour lies approximately along the 10 to 22 hour magnetic meridian.

\section{Distribution of Plasma Drift Velocities}

In the two following subsections, predictions of plasma flow patterns for the EISCAT and Sondre Stromfjord longitudes are presented as a function of geographic latitude and universal time. The latitude range is restricted to $10^{\circ}$ equatorward and poleward of each station's latitude. Horizontal plasma flow vectors are plotted at one degree latitude intervals and half hour UT intervals. The vectors are represented by straight lines, with a dot to represent the location at which the vector was calculated. This display format has been chosen for easy comparison between the Chatanika and Millstone Hill data and calculations (Sojka et al., 1979b). To highlight the three features listed in the introduction, broad solid lines and broad dashed lines have been included in the following figures to represent the polar region boundary and the reversal regions, respectively.

\section{EISCAT Predictions}

Figure 2 contains four panels; these correspond to the flow patterns associated with the four electric field models described earlier. EISCAT being a complex of two transmitters situated in Norway and three receiving stations, situated in Norway, Sweden and Finland, does not have a unique location. In the model calculations a geographic latitude of $70^{\circ}$ and a geographic longitude of $18^{\circ}$ were used for the EISCAT location. The EISCAT latitude is indicated by an arrow on the right-hand edge of each panel in Figure 2. A local time axis as well as a universal time axis are provided at the foot of Figure 2. For each model 90 kilovolts was used as the magnetospheric potential drop.

Panel 1 corresponds to the constant cross-tail electric field model, which was the model used in the earlier comparisons with Chatanika and Millstone Hill data (Sojka et al., 1979b). For more than half of the day the polar region boundary is within the EISCAT latitudinal range. The midnight flow reversal region and the equatorward part of the noon reversal are present. A large sector of the midnight polar region flow pattern can also be studied.

Panels 2 and 3 correspond to the asymmetric electric field models, with enhanced dusk and dawn magnetospheric electric fields, respectively. The resulting enhanced plasma flows in the polar regions can clearly be observed and differentiated from the constant cross-tail electric field model. Additionally, the time separation between the noon and midnight flow reversal regions changes significantly between these three panels. This time separation between features will provide a good indication of asymmetric polar region electric fields which in turn are indicative of changing interplanetary magnetic field directions (cf. Heppner, 1977; Fairfield, 1977; Stern, 1977). However, it should be noted that the convection patterns shown in Figure 2 were obtained by assuming a time-independent magnetospheric electric potential distribution.

Panel 4 shows the effects resulting from rotating the whole magnetospheric electric field by two hours. An overall time shift 


\section{EISCAT PREDICTIONS}
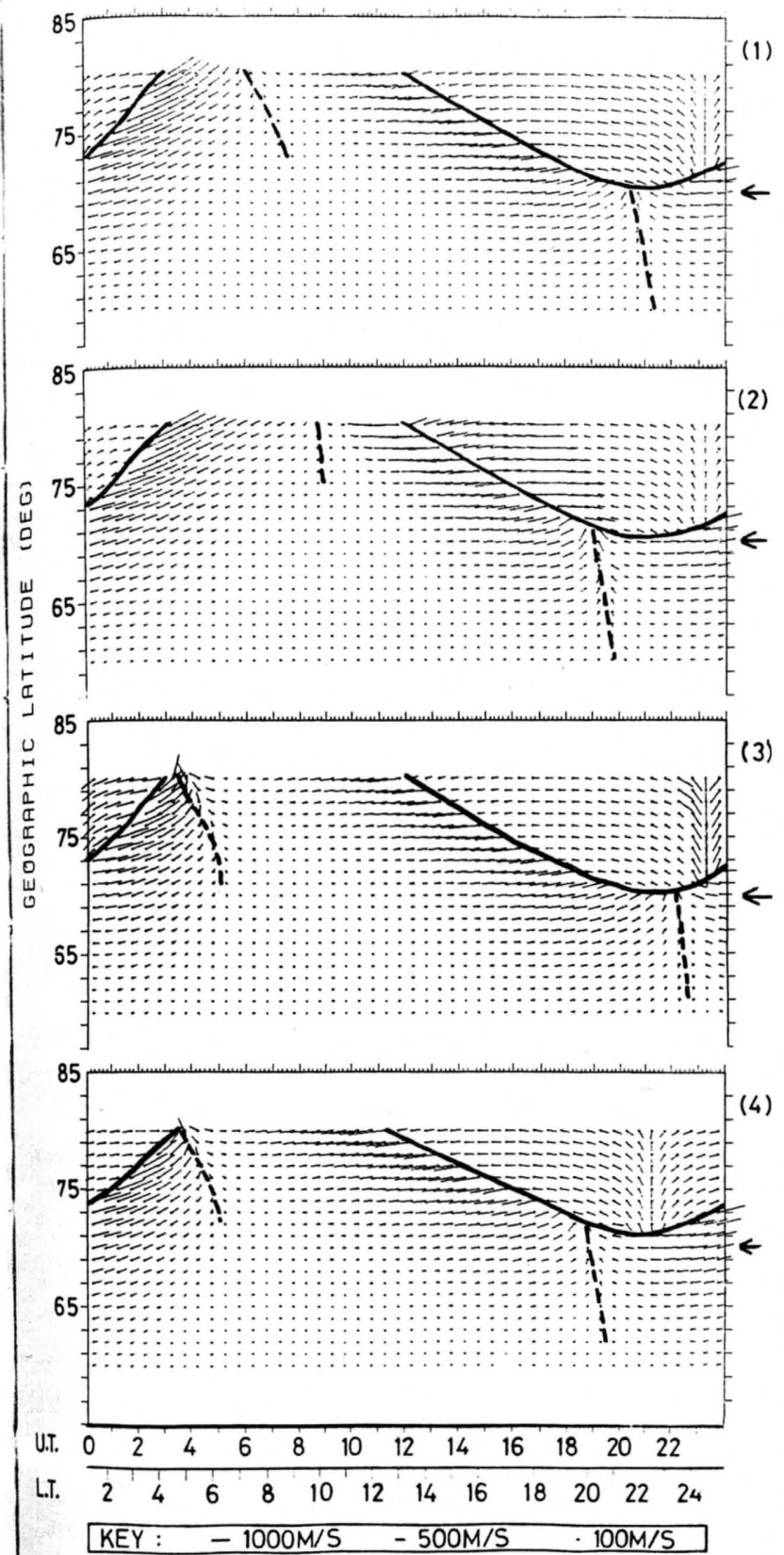

Fig. 2 Distribution of horizontal plasma drift velocities predicted for EISCAT for four different magnetospheric electric bield models. The top panel is for model (a) in Figure 1, the second and third panels are for asymmetric electric fields with enhancements on the dusk (model b) and dawn sides, respectively, and the bottom panel is for model (c).

of approximately two hours is found in the times at which the son and midnight flow reversals appear. Such a time phase shas actually present in both Chatanika and Millstone Hill (ata sets (Sojka et al., 1979b). However, these latter sites were thable to observe the associated polar region flow pattern, thich for this electric field model shows a significantly changed pattern (see panel 4).

\section{Pondre Stromfjord Predictions}

The geographic location of this site was taken to be $67^{\circ}$ latitude and $-51^{\circ}$ longitude. Figure 3 shows the four model predictions for this location. The display format is identical to that for Figure 2.

Panel 1 shows that the whole polar boundary region is in the field of view of Sondre Stromfjord for the case of a constant cross-tail electric field. However, only the noon flow reversal can be seen from this site. Unlike Chatanika, Millstone Hill and EISCAT, this site allows flow measurements to be made deep into the polar cap. Such measurements are particularly important in understanding the distribution of the electric field across the magnetosphere.

The effects of enhanced dusk (panel 2) and dawn (panel 3) magnetospheric electric fields is to produce large variations in the polar region plasma flow speeds. Enhanced flow regions extend over 1 to 2 hours of local time and hence should be easily identified when they occur. In addition, a marked change in the noon reversal region is associated with this asymmetry in the polar region electric field.

The rotation of the magnetospheric electric field causes a significant change in the polar region flow reversal pattern (panel 4). Such a change would be observable and is large enough to be contrasted with the other convection patterns. The local time change associated with the noon reversal region is comparable to that produced by the dawn asymmetric electric field (panel 3); however, the polar region flow patterns are quite different.

\section{Summary}

In this study we calculated diurnal patterns of plasma drift velocities which would be observed by EISCAT and by an incoherent scatter radar site at Sondre Stromfjord, Greenland. Four different magnetospheric electric field configurations were considered, including a constant cross-tail electric field, asymmetric electric fields with enhancements on the dawn and dusk sides of the polar cap, and an electric field pattern that is not aligned parallel to the noon-midnight magnetic meridian. The different electric field configurations produce signatures which are easy to identify in the plasma convection pattern, and hence, it should be possible to obtain valuable information on the magnetospheric electric field directly from convection patterns.

EISCAT and the Sondre Stromfjord site are particularly suited to studying three key features of the F-region convection pattern, i.e., the latitude of the midnight polar boundary, the local times of the noon and midnight flow direction reversals, and the latitude of the noon polar boundary as well as the flow pattern inside the noon polar region. These features cannot be studied adequately with existing radars, as all lie too far equatorward of the polar region.

The advantage of using these two sites, since neither is able to observe all three features, is that direct information concerning the magnetospheric electric field and how it maps into the ionosphere can be obtained. Measuring the local times at which flow reversal regions occur would differentiate between the relative importance of interplanetary magnetic field effects and a more general rotation of the field in the ionosphere. The variation of the latitude defining the polar region boundary enables the area into which the magnetospheric electric field is mapped to be defined, while detailed measurements of the flow pattern in this polar region would also indicate how the magnetospheric electric field is distributed. This whole region is known to vary with storm activity; however, these measurements would yield quantitative information on this variation in addition to the gross features of the high latitude plasma convection pattern.

In addition to using such key features to resolve different aspects of the magnetospheric electric field a number of other studies would be fruitful. Using the high sensitivity of EISCAT, measurements could be made of the flow pattern during quiet magnetospheric conditions. This study would be instructive, since the quiet time magnetospheric electric field is expected to contain fewer perturbing effects and would conse- 
SØNDRE STRØMFJORD PREDICTIONS

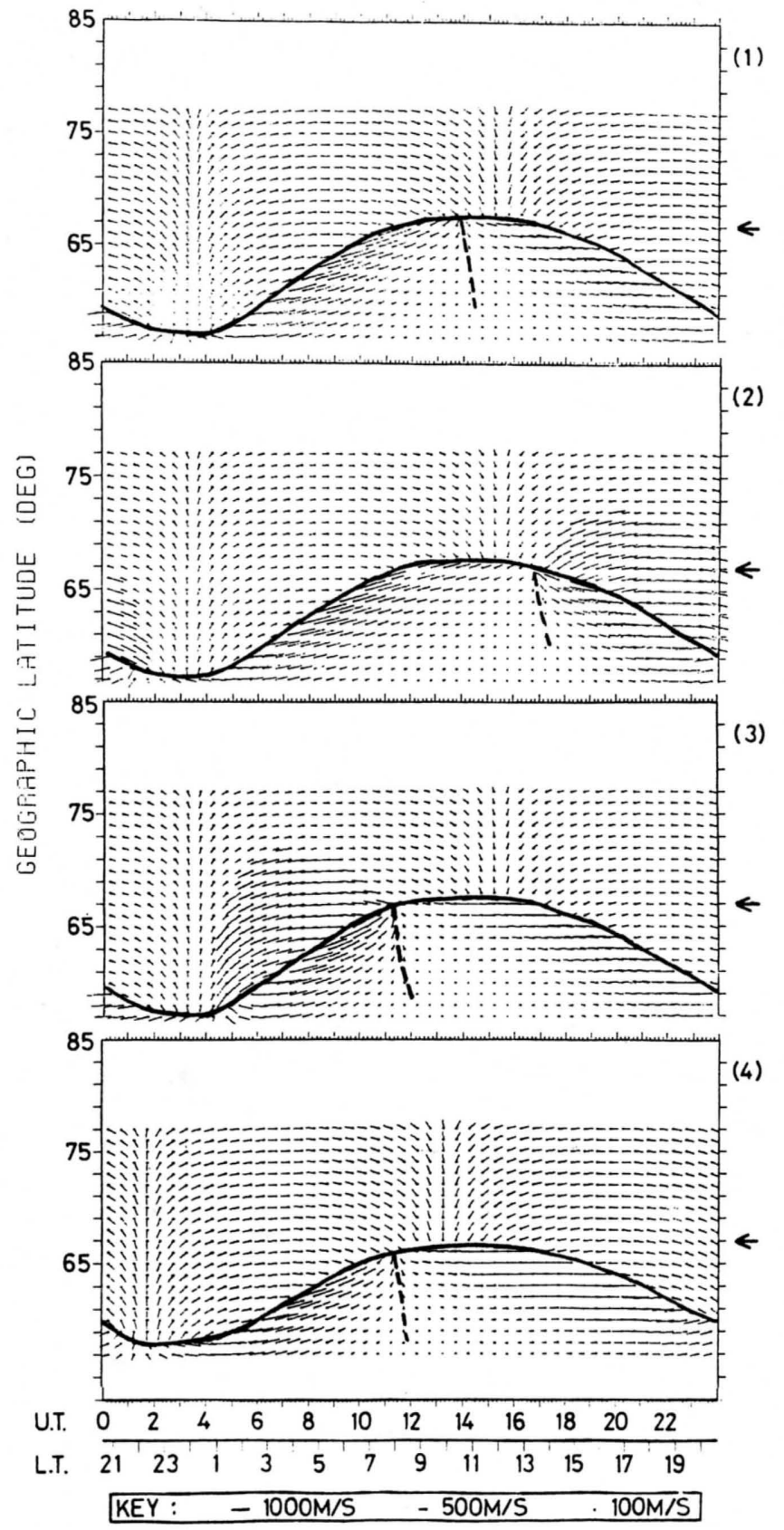
quently give the best information concerning the basic
magnetospheric electric field.

Finally, we summarize some of the more important assump. tions and limitations associated with our model predictions, First, we assumed a time-independent, magnetospheric electric potential distribution, and this potential distribution was mapped to an ionosphere which possessed a uniformly low elec trical conductivity distribution in the horizontal plane at F-region altitudes. We also assumed that the Earth's magnetic field could be adequately represented by an offset dipole, and we ignored the effects of substorms, which could occur several times a day and last for 2-3 hours. Nevertheless, with our simple convection model we have been able to obtain good agreement between model predictions and the different diurnal convection patterns observed simultaneously at Chatanika and Millstone Hill (Sojka et al., 1979b). We therefore are optimistic that our simple convection model is capable of predicting the gross pat. terns of plasma convection that will be observed by EISCAT and by a possible incoherent scatter radar facility located at Sondre Stromfjord, Greenland.

Acknowledgement. This research was supported by Air Force Contract USAF/ESD F19628-79-C-0025 and NSF Grant ATM78-10501 to Utah State University.

\section{References}

Fairfield, D.H. Electric and magnetic fields in the highlatitude magnetosphere, Rev. Geophys. Space Phys., 15, 285-298, 1977.

Heppner, J.P., Empirical models of high-latitude electric fields, J. Geophys. Res., 82, 1115-1125, 1977.

Mead, G.D., International geomagnetic reference field 1965.0 in dipole coordinates, J. Geophys. Res., 75, 4372, 1970.

Sojka, J.J., W.J. Raitt, and R.W. Schunk. Effect of displaced geomagnetic and geographic poles on high latitude plasma convection and ionospheric depletions. J. Geophys. Res., in press, 1979a.

Sojka, J.J., J.C. Foster, W.J. Raitt, R.W. Schunk, and J.R. Doupnik. High latitude convection: Comparison of a simple model with incoherent scatter observations. J. Geophys. Res., in press, $1979 \mathrm{~b}$.

Stern, D.P., Large-scale electric fields in the Earth's magnetosphere, Rev. Geophys. Space Phys., 15, 156-194 1977.

Yasuhara, F., Y. Kamide and S.-I. Akasofu, Field-aligned and

ionospheric currents, Planet. Space Sci., 23, 1355-1368, 1975.

(Received July 23, 1979; accepted August 20, 1979.)
Fig. 3 Distribution of horizontal plasma drift velocities predicted for Sondre Stromfjord for four different magnetospheric electric field models. The models are the same as for Figure 2 . 